\title{
Microfluidics for Single-Cell Genetic Analysis
}

\author{
A. M. Thompson ${ }^{a}$, A. L. Paguirigan ${ }^{b}$, J. E. Kreutz ${ }^{a}$, J. P. Radich ${ }^{b}$, and D. T. Chiua, ${ }^{\text {, }}$ \\ aDepartment of Chemistry, University of Washington, Seattle, WA, USA \\ ${ }^{b}$ Clinical Research Division, Fred Hutchinson Cancer Research Center, Seattle, WA, USA
}

\begin{abstract}
The ability to correlate single-cell genetic information to cellular phenotypes will provide the kind of detailed insight into human physiology and disease pathways that is not possible to infer from bulk cell analysis. Microfluidic technologies are attractive for single-cell manipulation due to precise handling and low risk of contamination. Additionally, microfluidic single-cell techniques can allow for high-throughput and detailed genetic analyses that increase accuracy and decreases reagent cost compared to bulk techniques. Incorporating these microfluidic platforms into research and clinical laboratory workflows can fill an unmet need in biology, delivering the highly accurate, highly informative data necessary to develop new therapies and monitor patient outcomes. In this perspective, we describe the current and potential future uses of microfluidics at all stages of single-cell genetic analysis, including cell enrichment and capture, single-cell compartmentalization and manipulation, and detection and analyses.
\end{abstract}

\section{Introduction}

The sequencing of the human genome through the Human Genome Project (HGP) was a seminal moment in biology. But like many great discoveries, it created even more questions and spurred research into areas of biology that were previously unknown. Work in proteomics, epigenetics, and posttranscriptional regulation, while significantly aided by the knowledge of the underlying genetic information, has demonstrated that the sequence of human genes alone is a basic framework onto which many layers of genetic regulation are applied. The disease-focused sequencing projects following the HGP, some of which capture multiple levels of genomic data such as The Cancer Genome Atlas, have enabled linking certain consistent genetic changes to specific diseases. However, it has also demonstrated that there is tremendous variation between individuals with similar diseases. Further research into the impact of this genetic information on disease has identified variation between cell populations within individuals. The ability to study this variation in depth will have significant implications for personalized medicine. Our knowledge of the extent to which intercellular variation plays a role in disease evolution and therapy outcome is currently limited by our inability to study small amounts of biological material, down to the level of an individual cell.

*Corresponding author: chiu@chem.washington.edu; Fax: + 1-206-685-8665; Tel: + 1-206-543-1665. 
Intra-sample heterogeneity likely holds valuable clues for understanding human disease and the variability between the responses of patients with the same disease to a given therapy. ${ }^{1}$ A clearer picture of how heterogeneity within individuals affects their disease progression and treatment can be a valuable tool for designing therapeutic regimens and defining treatments for different conditions. Perhaps turning an acute condition into a manageable, but chronic, one would be less risky than attempting to cure the individual entirely, especially in the case of therapies that involve alkylating agents or other potential mutationinducing treatments. Or perhaps, we might improve our ability to choose effective therapies for a given patient by adding to our understanding of the degree of heterogeneity in a patient's condition to risk-stratification criteria.

Over the last few decades, research methods for molecular analyses have improved in sensitivity and accuracy because of technology developed in a wide range of fields, from enzymology to microfluidics. This has resulted in the possibility of studying smaller quantities of starting material than traditionally used, along with huge increases in the density and types of data produced. Basic and clinical molecular research laboratories now have the ability to study a range of genetic material, from uncovering the identity and abundance of small RNAs via RNA sequencing to characterizing large chromosomal alterations via comparative hybridization arrays. The sensitivity increases in molecular techniques have also allowed us to identify the presence of low-frequency features that previously were not detectable. One issue hindering our ability to explore the biology of heterogeneous populations is that the amount of DNA or RNA required for most of the readily available in-depth genetic analysis methods are designed for bulk assays. These assays need on the order of nanograms or micrograms of material, which is a considerable amount given the minute content of a single cell for which the total available material is on the order of picrograms. Beyond total input issues, the question of isolating and handling single-cell materials without contamination or sample loss poses yet another hurdle for molecular analyses of heterogeneity at the single-cell level.

Probing genetic material at the level of a single cell will require new technologies to enhance capabilities and deliver accurate, actionable data for the wide range of questions being asked. Although new adaptations of macroscale methods are emerging to address these needs, the field of single-cell genetics requires a variety of fundamentally different strategies. Microfluidic technologies are in a unique position to address the limitations of current methods because they offer the benefits of both fluidic handling and thermal capabilities as well as flexibility in design, throughput and automation. In this perspective, we will discuss the scope and direction of scientific interests in single-cell genetics, highlight some of the ways microfluidics has proven useful in single-cell genetic analysis, and define areas where further improvement is needed.

\section{One cell, many questions}

Intra-sample genetic diversity, also known as clonal diversity, has diagnostic value in several diseases, such as predicting progression to malignancy in Barrett's esophagus. ${ }^{2}$ Clonal diversity has been demonstrated in breast cancer, ${ }^{3}$ and occurs in acute myeloid leukemia (AML)from diagnosis to relapse. ${ }^{4-6}$ Current strategies for estimating and tracking 
clonal diversity at the macroscale have used next-generation sequencing (NGS) of bulk tumor samples to determine the frequencies of mutant alleles. Changes in mutant allele frequencies can be observed over the course of therapy (comparing diagnosis to relapse), and between primary tumor sites and metastases. While these mutant alleles can be quantitatively tracked over time and over course of therapy in the bulk samples, the information about the specific disease-causing clone is lost in the background of all of the other cell types present in any patient sample. This is particularly challenging in samples where there is some ambiguity, such as in a biopsy of a tumor where the boundary of tumor verses normal tissue is not completely clear or in a peripheral blood sample where the amount of leukemic cells varies between patients.

Rather than attempting to infer concurrent occurrence of different genetic characteristics seen in averaged data from a bulk sample, being able to assess the genomic or expression characteristics of individual cells themselves can directly link genotype and expression data that occur simultaneously in a cell. If a cell with a specific set of mutations doesn't actually express those alleles, or has other downstream regulatory changes that cause a different set of targets to be expressed or inhibited, targeting that pathway would incorrectly destroy the wrong cell types and potentially allow the rogue cell to continue to proliferate and cause relapse of disease. RNA and DNA extracted from bulk samples does provide a general description of the population average in the original sample, but it is impossible to reconstruct how the different populations may have contributed to that average. One can find correlations, for example, between mutational allele frequencies and the level of expression of RNAs downstream of that gene, but even this information does not inform whether these events occur concurrently in the same cells. When a population average is measured, the technique used requires a relatively large amount of starting material to ensure there is enough to avoid sampling issues and stochastic variability in the results. These methods often are not validated at the small amounts that would make integration with single-cell assays accurate or reasonable.

Additionally, the separation of measurement or technical variability from biological variability in each measurement platform can be challenging but is crucial for the validation of any single-cell assay where analyte amounts are near the limit of detection. Normalization strategies typically used in bulk measurements are not appropriate for single cells (i.e., technical variability in control genes during a qPCR experiment would cause normalization of the target gene measurement to be erroneous).For this reason, having suitable controls becomes an issue for validation of single-cell data. Another challenge unique to single-cell molecular analyses is the issue of total sample size (or total cell number analyzed per tissue sample). As for the degree of heterogeneity in a sample, when the technical variability inherent to the assay and the number of parameters analyzed increases, the number of cells that need to be analyzed to describe the overall heterogeneity of a sample with statistical significance must rapidly increase. Available methods for the physical isolation and handling of individual cells for emerging and sensitive genetic analysis techniques limit sample size because of their low throughput, high cost per cell, or high failure rates.

Currently, there are few powerful tools readily available to identify heterogeneity at the single-cell level. For decades we have been able to process very large populations (millions 
of cells analyzed per sample), and quickly identify frequencies of different cell types within a single sample by using a wide range of cell surface markers or intracellular stains. For example, flow cytometry is by far the most rapid, complex (multi-parameter) and immediate (protein-based) data producing tool available for single-cell analysis. However, molecular genetic analyses of single cells (both genomic and gene expression) has not had the benefit of decades of tools developed to analyze multiple features simultaneously in individual cells with high enough throughput or data complexity (multiple parameter data). Translating the discovery tools that are effective for bulk samples, such as large scale sequencing and other genetic analysis methods, to the single-cell level will be invaluable to further elucidate mechanisms of disease and how individual cells make choices and regulate their various processes.

\section{Microfluidics as a solution}

New methods for single-cell assays must provide the means to link genetic data to an individual cell's characteristics as well as address the major limitations for effective analysis. Specifically, they must: 1) provide the handling precision necessary to isolate and manipulate minute quantities of biological material, 2) approach single-molecule sensitivity to eliminate bias due to amplification, 3) provide high accuracy as the same cell cannot be measured multiple times, 4) provide throughput high enough to efficiently generate statistically meaningful data, and 5) eliminate contamination from the environment and components within the sample. For a method to be successfully adopted into research and clinical settings, ease of use, integration with existing infrastructure, and cost are critical factors. Microfluidics has shown strong performance in these areas outside of the genetics arena. Research incorporating microfluidics and single-cell genetic analysis, including cell capture and enrichment, cell compartmentalization, and detection can be used to create simple and more informative tools for single-cell study. Specific advantages to applying a microfluidic approach to this complex field are outlined in Figure 1 and highlighted in the remainder of this paper.

\section{Capture and enrichment of single cells}

Correlation of genetic data with its single cell of origin requires a method to isolate single cells from a tissue. Currently, methods for the selection and transfer of single cells into wells or tubes include laser capture microdissection, optical tweezer manipulation, micromanipulation, flow cytometry, or microfluidic methods. These methods differ in their equipment requirements, cost, degree of user skill, tissue compatibility, and throughput. Flow cytometry is attractive because of its multiparameter sorting and high throughput, but depositing cells into microliter volume wells results in dilution of analytes and does not allow the user to easily confirm that cells were deposited successfully into the analysis volume. Laser capture microdissection can provide certainty of cell isolation, but at very low throughput. ${ }^{7}$ Enrichment and compartmentalization within a microfluidic platform can be designed for high throughput while minimizing dilution and contamination risk. A number of microfluidic techniques have been developed to address these needs.

A variety of techniques have been used to indiscriminately trap single cells from a cell suspension in microfluidic systems. These methods include hydrodynamic mechanisms, or 
use electrical, optical, magnetic or acoustic fields to control trapping. An extensive review of cell trapping methods is presented by Nilsson et al. ${ }^{8}$ To be integrated with downstream genetic analysis, these devices must be compatible with isolation, manipulation, and analysis or retrieval mechanisms. The commercial microfluidic C1 Single-Cell Auto Prep System form Fluidigm uses hydrodynamic capture and isolation of single cells from suspension before cell lysis and processing single-cell genetic material before retrieval and use with multiplex PCR, RT-PCR, or NGS methods. A disadvantage of this and other hydrodynamic trapping devices is that an excess of cells are needed for high trapping density, resulting in a loss of the majority of the single cells from the incoming sample. These methods also are unable to select specific types of cells in a sample, even those types that are fairly common. For many single-cell applications, a simple, straightforward device for trapping thousands of single cells, rather than hundreds, would expand the studies that could be executed with statistically significant data.

Some questions regarding single cells are focused on assessing genetic heterogeneity in only a small sub-population of single cells in a biological sample. Rare cells, usually thought of as having cellular abundance less than 0.1 percent, are relevant markers in cancer, prenatal diagnosis, and infectious disease. In order to gather sufficient data from patient samples, single-cell enrichment techniques typically must offer high sample throughput and yield high recoveries of target cells. If these enrichment devices are to be used upstream of cell trapping, isolation, manipulation and genetic analysis methods, the techniques should allow for high purity retrieval of viable cells. Circulating tumor cells (CTCs), an extraordinarily rare cell type, are present in quantities near one cell per 1 billion blood cells in patients with advanced stage cancer. Methods such as Fluorescence Activated Cell Sorting (FACS), Magnetic Activated Cell Sorting (MACS), and cell affinity separations are high-throughput and very effective for some applications, but they typically have low-recovery efficiency for CTCs and will deposit $0.1 \%$ of background cells with the target population. This background noise limits their applicability in cases such as genetic analysis of single CTCs. ${ }^{9}$ In contrast, microfluidic mechanisms for isolation of CTCs have been demonstrated that enrich these rare cells with high recovery. These techniques utilize laminar flow and/or high surface-to-volume ratios to operate. For example, antibodies can be used to immobilize specific cell types in $\operatorname{static}^{10}$ (Fig 2a) or flow ${ }^{11}$ (Fig 2b)systems, optical methods can be combined with valves to create CTC-containing aliquots of sample ${ }^{12}$ (Fig 2c), and fabricated filters and flow focusing can be integrated upstream of labelled CTC separation and collection by magnetophoresis ${ }^{13}$ (Fig $2 \mathrm{~d}$ ).

\section{Compartmentalization}

On its own, trapping cells is insufficient for single-cell analysis because the contents of those cells, once lysed, aren't necessarily isolated from each other. The cells also have to be compartmentalized in such a way as to maintain all biological materials from a single cell in an isolated fluidic space that is separate from other cells. There are a variety of methods that have been developed for the compartmentalization of cells for downstream analysis (Fig 3). Valve-based systems can pneumatically isolate cells, and often utilize traps upstream of the sample handling (Fig 3A). ${ }^{14}$ Droplet generation systems also can be used to isolate cells, without the need for traps or valves, which drastically can simplify device design/ 
fabrication. These droplet systems also have the potential for much higher throughput than valve-based systems. However, droplet platforms typically rely on partitioning of cells into droplets following a Poisson distribution, which can limit throughput due to a large number of droplets being empty and a few with two or more cells. If throughput is high enough, a sufficient rate of single-cell measurements can be obtained despite these uninformative droplets. Techniques also exist to selectively encapsulate single cells into individual droplets (Fig 3B) $)^{15}$ or to "beat" Poisson statistics (Fig 3C). ${ }^{16}$

Another approach is to isolate cells in chambers, but without using valves. Typically systems that isolate chambers (containing cells, beads, or some other component) have the sample fully fill the device and then cap the chambers with an oil or a physical barrier. ${ }^{17-18}$ Other systems are essentially extensions of droplet platforms, but keep the volumes isolated using physical barriers rather than emulsion stabilizing surfactants. This maintains some of the advantages of droplet systems (reduced risk of fouling of the device surface and crosstalk), while facilitating the tracking of individual samples over an extended time period. Examples of systems that enable additional sample processing include the SlipChip, ${ }^{19}$ SD chip, ${ }^{20}$ some valve-based chips, ${ }^{21}$ and some hybrid emulsion/physical isolation systems. ${ }^{22}$

It is relevant to note that by isolating single cells into compartments, intercellular interactions and any effects of the native cell matrix on the genome are removed. Methods to probe such interactions in a controlled environment, through arrays that incorporate cell culture or media exchange prior to isolation and lysis, for instance, would both enhance our understanding of cellular processes and might validate the results of techniques studying cells in isolation. A recent review of microfluidic devices to probe cell-cell communication is given by Guo et al. ${ }^{23}$

Once isolated, performing cell lysis in these individual, microfluidic compartments minimizes exposure to contamination from other cells within the sample or from material in the laboratory. Lysis methods should preserve the integrity of the genetic material and chemical lysis methods should be compatible with downstream enzymatic reactions such as PCR. Also, stress induced cell-signalling that might alter transcript levels should be avoided. Methods for cell lysis include physical, chemical, thermal, and electrical techniques with varying lengths of time to lyse the cell and different design requirements for the microfluidic device. Each of these techniques have been utilized in microfluidic nucleic acid analysis devices reviewed by Kim et al. ${ }^{24}$

\section{Analysis of single-cell genetic material}

Analyzing material from a single cell is challenging in many regards. In the single cell, RNA is present in picrogram quantities; some low abundance RNA transcripts are present in 1-10 copies. ${ }^{7}$ While qPCR and qRT-PCR theoretically are able to amplify and detect singlemolecule quantities of nucleic acids, it is challenging to quantify low quantities in microliter-volume reactions. For one, amplification bias causes copy number uncertainty. ${ }^{15}$ Additionally, the qPCR signal is analog, requiring "real time" monitoring, and signal calibration ${ }^{25}$ or internal reference standards for relative gene expression that are complicated by the stochastic nature of gene expression at the single-cell level. ${ }^{26}$ Whole-genome or 
whole-transcriptome amplification is requisite for single-cell analysis using NGS platforms. But in the process of amplification, information about the spatial arrangement of sequences, copy number variation, or relative gene expression are not fully conserved due to variations in amplification efficiency and transcript length limitations. Additionally, while NGS provides a huge amount of data per cell, it is currently cost-prohibitive to perform NGS on sufficient numbers of cells to describe a population or to describe the contribution of measurement/technical error in any statistically relevant way.

Digital PCR and RT-PCR-Digital PCR is one way to count individual gene molecules without the need for a calibration curve or a normalization gene (Fig 4). For these assays, the analysis volume is discretized such that the analyte is randomly distributed into many small volumes before gene and signal amplification and detection. The number of volumes analyzed per cell should be high enough so that some volumes do not contain the target analyte, and the Poisson distribution can be applied to calculate the likelihood of multiple targets occupying the same droplet. PCR is then performed in the discrete volumes.

Endpoint detection can be used to calculate the initial concentration based on the assumption of a random distribution of starting analytes into the assay volumes. Microfluidic devices for this technique achieve high copy number precision and dynamic range by rapidly generating discrete, known volumes from the target samples. Performing these analyses in individually addressable volumes may also allow for further downstream analysis(Fig 4A). ${ }^{27}$ As an alternative to high droplet capacity devices, Kreutz el al. showed that a wide dynamic range and high copy number resolution can be achieved by using a smaller number of chambers and multivolume digital PCR (Fig 4B). ${ }^{29}$

Despite improvements in the accuracy, sensitivity, and reproducibility of digital PCR for the quantification of DNA, gene expression analysis has not achieved the same performance standards. Digital RT-PCR requires a reverse-transcription step for the construction of a cDNA library before preamplification and digitization into microfluidic volumes. This reverse transcription step is known to suffer from variations in efficiency between transcripts. ${ }^{25-26,30}$ Variations in sample preparation steps result in vastly different results in digital RT-PCR, ${ }^{25}$ although with consistent sample preparation, results may be highly reproducible between measurements. ${ }^{30}$ Performing reverse-transcription and preamplification steps in microfluidic volumes may offer some reduction in amplification bias inmicroliter-volume reactions. In one study comparing various methods of single-cell preparation, performing these enzymatic reactions in nanoliter, rather than microliter, volumes resulted in the best correlation between quantitative sequencing and PCR results. ${ }^{30}$

Digitization of the sample volume derived from a single cell can also be useful for preserving haplotype information. However, information about co-localization of mutations on a single chromosome is lost because of limitations in product length that result from enzymatic nucleic-acid amplification. Fan et al. showed that by using a microfluidic device to compartmentalize the individual chromosomes of a single cell, this information is preserved during downstream analysis (Fig 4C). ${ }^{17}$

Microfluidic PCR—Digital PCR typically focuses on 1-2 targets per sample. But in order to draw statistically meaningful conclusions about gene expression heterogeneity in tissues, 
it is necessary to analyze a larger number of cooperating targets from a large number of single cells. The complex and rapid sample handling procedures possible with microfluidics provide a platform for such high-throughput gene expression analysis of single cells with the possibility to tease out measurement/technical variability from biological variability. Using the Dynamic Array integrated fluidic circuits developed by Fluidigm, it is possible to simultaneously measure gene expression of 96 gene targets in 96 single cells. ${ }^{7}$ This method uses single-cell isolation by flow cytometry or laser capture microdissection but similar preparations can be performed by using the recently available microfluidic $\mathrm{C}_{1}{ }^{\mathrm{TM}}$ Single-Cell Auto Prep System (Fluidigm). Realistically, these methods, despite being more data-dense than previous ones, still are limited by total system cost per cell, overall throughput (larger number of cells are required for statistical power) and are currently challenging to integrate with laboratory infrastructure.

Sample preparation for next-generation sequencing-NGS methods produce reads from single nucleic acid molecules in a high-throughput fashion, and thus require wholegenome amplification or reverse transcription and whole transcriptome amplification. Whole-genome amplification is often carried out using multiple displacement amplification (MDA), but, depending on the biological sample, this method has been demonstrated to have varying levels of amplification bias. Marcy et al. showed that reducing the volume of single bacterial cell MDA reactions from $50 \mu \mathrm{L}$ to $60 \mathrm{~nL}$ reactors produced more specificity and greater amplification uniformity. ${ }^{31}$ Although improvements in sample preparation have helped the completeness and accuracy of the reverse transcription and preamplification steps, current RNA-sequencing methods cannot be considered as absolute counting technologies. ${ }^{32}$ Recently, Wu et al. compared the sensitivity and reproducibility of typical single-cell whole transcriptome preparations. They found that performing reverse transcription and preamplification steps in microfluidic volumes of the $\mathrm{C} 1$ device (Fluidigm), rather than tube-based preparations, produced less gene dropout, improved reproducibility (defined as deviations about the sample mean), and accuracy (defined by comparing gene expression levels to those calculated by single-cell qPCR). ${ }^{30}$

Single-molecule techniques-Despite improvements in whole-genome and whole transcriptome amplification techniques, the error inherent to these methods continues to limit the reliability of NGS, and to a lesser extent, digital and multiplex PCR. Methods of directly counting single molecules to avoid amplification and reverse transcription of singlecell genetic material would be highly valuable. Other single-molecule nucleic acid counting techniques include fluorescent labelling techniques such as single-molecule $\mathrm{FISH}^{26}$ and Nanostring's nCounter system. ${ }^{33}$ In single-molecule FISH, multiple fluorescent probes bind to each mRNA, and spots are counted using fluorescence microscopy. The method has been demonstrated on both fixed and live cells, the latter made possible by using probes that can be transported through the living cell membrane. ${ }^{26}$ While the use of microfluidics is not necessary to perform single-molecule FISH, using the method in combination with microfluidic cell compartmentalization could automate image acquisition and analysis. ${ }^{34}$ 


\section{Future outlook}

Despite improvements in single-cell genetic analysis capabilities, further improvements in single-cell handling, enrichment, and analysis techniques are necessary for these methods to make an impact on our understanding of biology. There are a number of needs that are priorities for generating a strong set of single-cell data for human biology research. Microfluidic device designs that address these issues early on in their development will be far more likely to allow researchers to access a broader range of single-cell characteristics in a statistically meaningful way.

Strategies for manipulation and interrogation of single cells should aim to improve upon information accuracy, amount of information obtained per cell, and single-cell throughput. Performing whole genome amplification in small volumes has already been shown to better preserve relative gene abundance for more accurate gene quantification, and future analysis systems requiring whole genome amplification should continue to use microfluidic volumes for these operations. Accuracy of single-cell genetic analysis systems will also be improved if whole genome amplification is limited or avoided, which may be possible using innovative single-molecule detection strategies that take advantage of minimal dilution offered by microfluidic systems. Future methods to increasing the amount of information per cell might come in the form of incorporating increasingly accurate whole genome amplification with highly informative NGS. The ability to integrate multiple manipulation operations and analytical detection strategies on a single microfluidic device could also lead to complex systems generating data on multiple gene targets or multiple macromolecule types. Currently, the throughput of microfluidic single-cell genetic analysis systems has been limited to hundreds single-cells. Future microfluidic designs should explore avenues to decrease the number of cells wasted during trapping and compartmentalization, increase the density of single cell arrays, and ultimately increase the number of single cells analysed per device. As always, ease of use, cost, and analysis time should be considered for any technology moving towards commercialization.

Beyond the platforms employed for isolating and analyzing single cells, additional computational methods will be crucial for researchers to address technical variation and identify the degree of significance of any biological variation detected. Large data sets (as from single-cell sequencing data sets), ${ }^{35}$ and smaller, more focused data sets (as from digital PCR and RT-PCR assays), ${ }^{36}$ will need slightly different validation strategies. Ideally, the integration of multiple data types originating from the same single cells will be possible (for example, cell surface markers with gene expression with genotyping). The generation and curation of single-cell data sets from both normal and diseased human tissues would provide a valuable understanding of the types of variation that are normal in human development and those that are hallmarks of disease evolution and progression.

\section{Supplementary Material}

Refer to Web version on PubMed Central for supplementary material. 


\section{Acknowledgements}

We gratefully acknowledge support from the National Institutes of Health (R01CA175215).

\section{References}

1. Wills QF, Livak KJ, Tipping AJ, Enver T, Goldson AJ, Sexton DW, Holmes C. Nat. Biotechnol. 2013; 31:748. [PubMed: 23873083]

2. Merlo LM, Shah NA, Li X, Blount PL, Vaughan TL, Reid BJ, Maley CC. Cancer Prev Res (Phila). 2010; 3:1388-1397. [PubMed: 20947487]

3. Park SY, Gonen M, Kim HJ, Michor F, Polyak K. J Clin Invest. 2010; 120:636-644. [PubMed: 20101094]

4. Ding L, Ley TJ, Larson DE, Miller CA, Koboldt DC, Welch JS, Ritchey JK, Young MA, Lamprecht T, McLellan MD, McMichael JF, Wallis JW, Lu C, Shen D, Harris CC, Dooling DJ, Fulton RS, Fulton LL, Chen K, Schmidt H, Kalicki-Veizer J, Magrini VJ, Cook L, McGrath SD, Vickery TL, Wendl MC, Heath S, Watson MA, Link DC, Tomasson MH, Shannon WD, Payton JE, Kulkarni S, Westervelt P, Walter MJ, Graubert TA, Mardis ER, Wilson RK, DiPersio JF. Nature. 2012; 481:506-510. [PubMed: 22237025]

5. Jan M, Snyder TM, Corces-Zimmerman MR, Vyas P, Weissman IL, Quake SR, Majeti R. Sci. Transl. Med. 2012; 4:149ra118.

6. Welch JS, Ley TJ, Link DC, Miller CA, Larson DE, Koboldt DC, Wartman LD, Lamprecht TL, Liu F, Xia J, Kandoth C, Fulton RS, McLellan MD, Dooling DJ, Wallis JW, Chen K, Harris CC, Schmidt HK, Kalicki-Veizer JM, Lu C, Zhang Q, Lin L, O'Laughlin MD, McMichael JF, Delehaunty KD, Fulton LA, Magrini VJ, McGrath SD, Demeter RT, Vickery TL, Hundal J, Cook LL, Swift GW, Reed JP, Alldredge PA, Wylie TN, Walker JR, Watson MA, Heath SE, Shannon WD, Varghese N, Nagarajan R, Payton JE, Baty JD, Kulkarni S, Klco JM, Tomasson MH, Westervelt P, Walter MJ, Graubert TA, DiPersio JF, Ding L, Mardis ER, Wilson RK. Cell. 2012; 150:264-278. [PubMed: 22817890]

7. Sanchez-Freire V, Ebert AD, Kalisky T, Quake SR, Wu JC. Nat. Protoc. 2012; 7:829-838. [PubMed: 22481529]

8. Nilsson J, Evander M, Hammarstrom B, Laurell T. Anal. Chim. Acta. 2009; 649:141-157. [PubMed: 19699390]

9. Gao Y, Li W, Pappas D. Analyst. 2013; 138:4714-4721. [PubMed: 23778244]

10. Hou S, Zhao H, Zhao L, Shen Q, Wei KS, Suh DY, Nakao A, Garcia MA, Song M, Lee T, Xiong B, Luo SC, Tseng HR, Yu H-h. Adv. Mater. 2013; 25:1547-1551. [PubMed: 23255101]

11. Dharmasiri U, Njoroge SK, Witek MA, Adebiyi MG, Kamande JW, Hupert ML, Barany F, Soper SA. Anal. Chem. 2011; 83:2301-2309. [PubMed: 21319808]

12. Schiro PG, Zhao M, Kuo JS, Schneider T, Koehler KM, Sabath DE, Chiu DT. Angew. Chem. Int. Ed. 2012; 51:4618-4622.

13. Ozkumur E, Shah AM, Ciciliano JC, Emmink BL, Miyamoto DT, Brachtel E, Yu M, Chen P, Morgan B, Trautwein J, Kimura A, Sengupta S, Stott SL, Karabacak NM, Barber TA, Walsh JR, Smith K, Spuhler PS, Sullivan JP, Lee RJ, Ting DT, Luo X, Shaw AT, Bardia A, Sequist LV, Louis DN, Maheswaran S, Kapur R, Haber DA, Toner M. Sci. Transl. Med. 2013; 5:179ra47.

14. White AK, Vanlnsberghe M, Petriv OI, Hamidi M, Sikorski D, Marra MA, Piret J, Aparicio S, Hansen CL. PNAS. 2011; 108:13999-14004. [PubMed: 21808033]

15. He M, Edgar JS, Jeffries GDM, Lorenz RM, Shelby JP, Chiu DT. Anal. Chem. 2005; 77:15391544. [PubMed: 15762555]

16. Edd JF, Carlo DD, Humphry KJ, Köster S, Irimia D, Weitz DA, Toner M. Lab Chip. 2008; 8:1262-1264. [PubMed: 18651066]

17. Fan CH, Wang J, Potanina A, Quake SR. Nat. Biotechnol. 2011; 29:51-56. [PubMed: 21170043]

18. Rissin DM, Kan CW, Campbell TG, Howes SC, Fournier DR, Song L, Piech T, Patel PP, Chang L, Rivnak AJ, Ferrell EP, Randall JD, Provuncher GK, Walt DR, Duffy DC. Nat. Biotechnol. 2010; 28:595-599. [PubMed: 20495550]

19. Du WB, Li L, Nichols KP, Ismagilov RF. Lab Chip. 2009; 9:2286-2292. [PubMed: 19636458] 
20. Cohen DE, Schneider T, Wang M, Chiu DT. Anal. Chem. 2010; 82:5707-5717. [PubMed: 20550137]

21. Leung K, Zahn H, Leaver T, Konwar KM, Hanson NW, Pagé AP, Lo CC, Chain PS, Hallam SJ, Hansen CL. PNAS. 2012; 109:7665-7670. [PubMed: 22547789]

22. Schmitz CHJ, Rowat AC, Köster S, Weitz DA. Lab Chip. 2009; 9:44-49. [PubMed: 19209334]

23. Guo F, French JB, Li P, Zhao H, Chan CY, Fick JR, Benkovic SJ, Huang TJ. Lab Chip. 2013; 13:3152-3162. [PubMed: 23843092]

24. Kim J, Johnson M, Hill P, Gale BK. Integr. Biol. 2009; 1:574-586.

25. Sanders R, Mason DJ, Foy CA, Huggett JF. PLoS One. 2013

26. Raj A, van Oudenaarden A. Annu. Rev. Biophys. 2009; 38:255-270. [PubMed: 19416069]

27. Schneider T, Yen GS, Thompson AM, Burnham DR, Chiu DT. Anal. Chem. 2013; 85:1041710423. [PubMed: 24099270]

28. Gansen A, Herrick AM, Dimov IK, Lee LP, Chiu DT. Lab Chip. 2012; 12:2247-2254. [PubMed: 22399016]

29. Kreutz JE, Munson T, Huynh T, Shen F, Du W, Ismagilov RF. Anal. Chem. 2011; 83:8158-8168. [PubMed: 21981344]

30. Wu AR, Neff NF, Kalisky T, Dalerba P, Treutlein B, Rothenberg ME, Mburu FM, Mantalas GL, Sim S, Clarke MF, Quake SR. Nat. Methods. 2014; 11:41-46. [PubMed: 24141493]

31. Marcy Y, Ishoey T, Lasken RS, Stockwell TB, Walenz BP, Halpern AL, Beeson KY, Goldberg SM, Quake SR. PLoS Genet. 2007; 3:1702-1708. [PubMed: 17892324]

32. Picelli S, Bjorklund AK, Faridani OR, Sagasser S, Winberg G, Sandberg R. Nat. Methods. 2013; 10:1096-1098. [PubMed: 24056875]

33. Geiss GK, Bumgarner RE, Birditt B, Dahl T, Dowidar N, Dunaway DL, Fell HP, Ferree S, George RD, Grogan T, James JJ, Maysuria M, Mitton JD, Oliveri P, Osborn JL, Peng T, Ratcliffe AL, Webster PJ, Davidson EH, Hood L, Dimitrov K. Nat. Biotechnol. 2008; 26:317-325. [PubMed: 18278033]

34. Matsunaga T, Hosokawa M, Arakaki A, Taguchi T, Mori T, Tanaka T, Takeyama H. Anal. Chem. 2008; 80:5139-5145. [PubMed: 18537270]

35. Yilmaz S, Singh AK. Curr. Opin. Biotechnol. 2012; 23:437-443. [PubMed: 22154471]

36. McDavid A, Finak G, Chattopadyay PK, Dominguez M, Lamoreaux L, Ma SS, Roederer M, Gottardo R. BMC Bioinformatics. 2013; 29:461-467. 


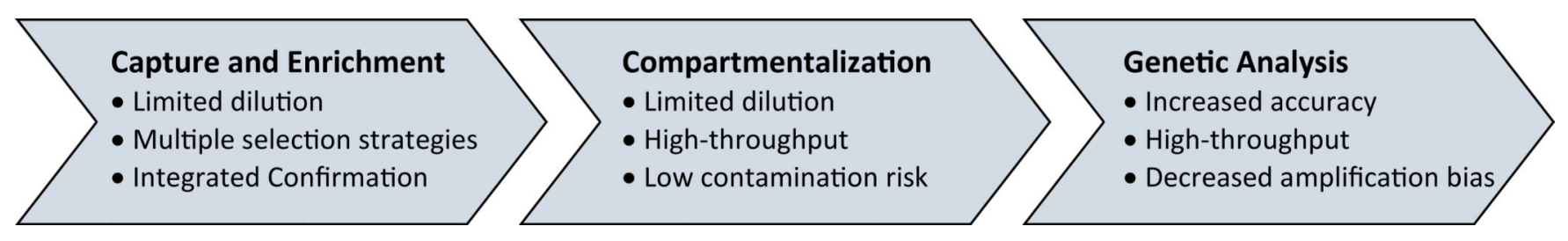

Fig. 1.

Advantages of microfluidics for single-cell genetic analysis. Microfluidics technologies offer advantages at various stages of single-cell genetic analysis. In this paper, the current and future applications of microfluidics to provide simple and informative analyses in this field are discussed. 
A)

C) CTC capture at $37^{\circ} \mathrm{C}$

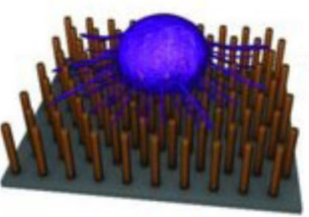

Cool down $\underset{\text { Warm up }}{\rightleftarrows}$ Tar
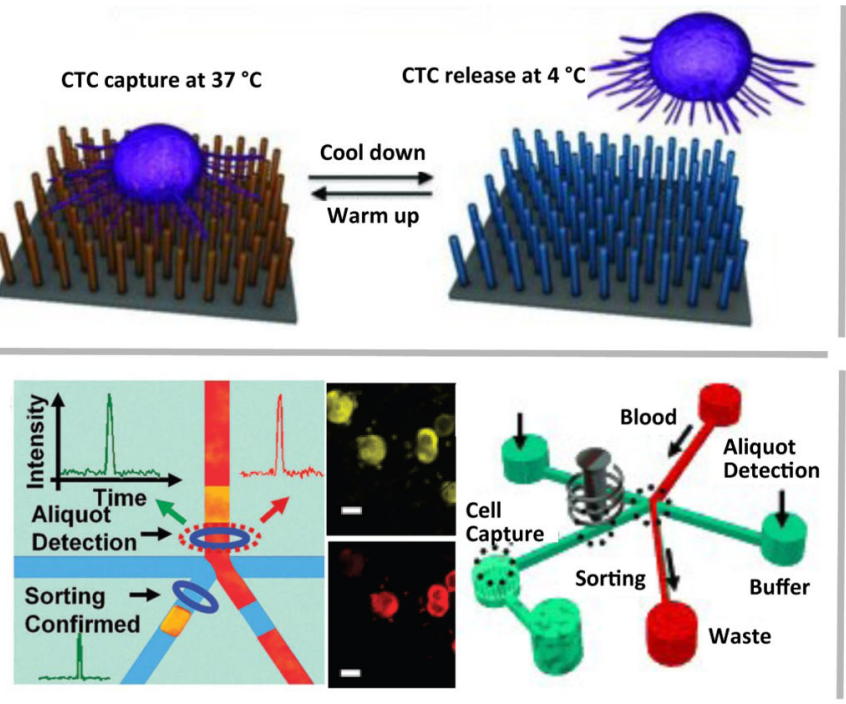

B)
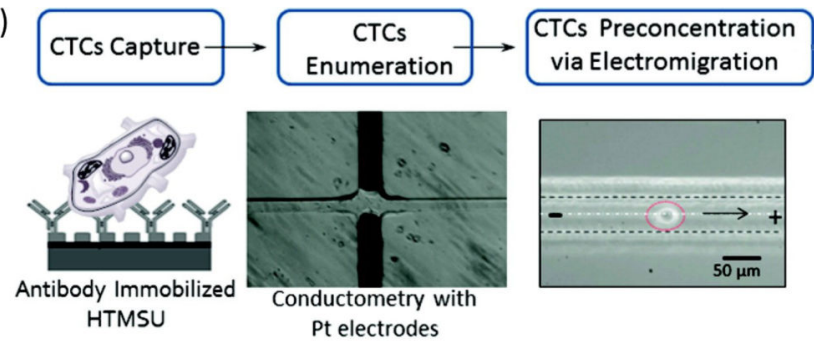

HTMSU

Pt electrodes

D) Hydrodynamic cell sorting $\rightarrow$ Inertial Focusing $\rightarrow$ Magnetophoresis

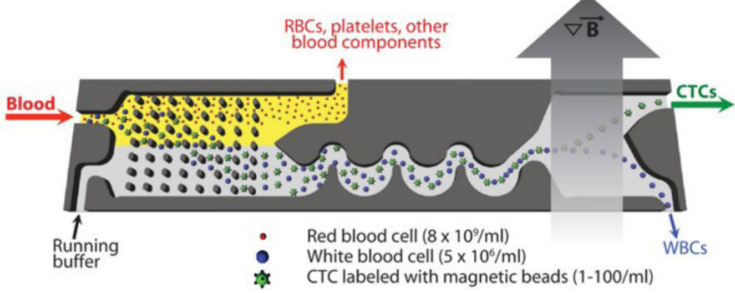

Fig. 2.

Microfluidic enrichment of rare cells. Circulating tumor cells (CTCs) can be enriched from whole blood using a variety of techniques including A) nanostructure and antibody-based reversible surface capture [reprinted (adapted) with permission from ref. 10. Copyright 2013 Nature Publishing Group], B) affinity capture combined with electrokinetic enrichment [reprinted (adapted) with permission from ref. 11. Copyright 2011 American Chemical Society], C) microfluidic aliquot sorting of target cells combined with on-chip filtration Reprinted (adapted) with permission from ref. 12. Copyright 2012 Angewandte Chemie, International Edition], and D) combined hydrodynamic-magnetic methods Reprinted (adapted) with permission from ref. 13. Copyright 2013 AAAS]. 
A)

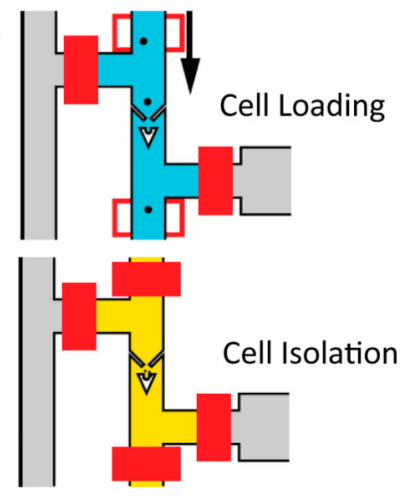

Empty Cell Suspension Wash Buffer

Closed Valve $\square$ Open Valve
B)
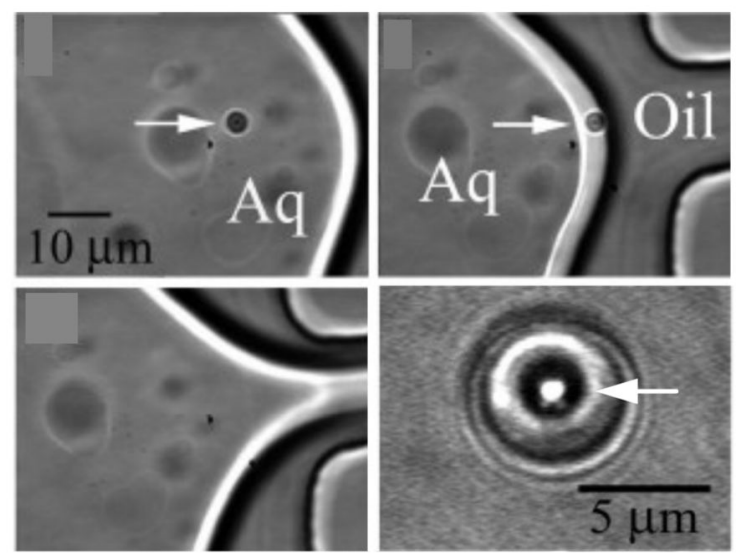

C) Random (Poisson) encapsulation
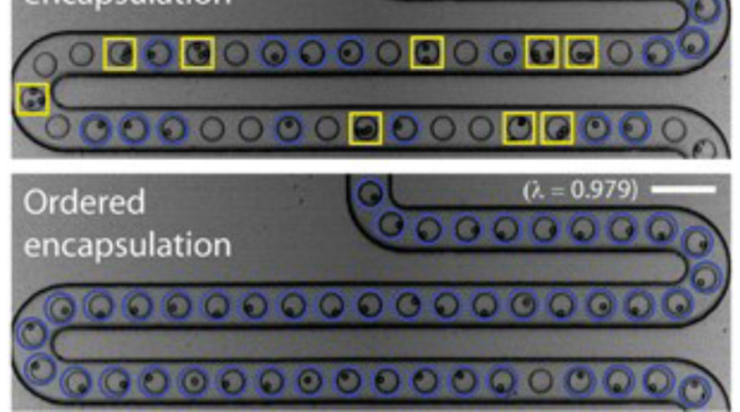

Fig. 3.

Compartmentalization of single cells. Numerous microfluidic methods have been developed to form discrete aqueous volumes to spatially confine aqueous volumes on-chip or to trap single cells. A) A hydrodynamic single-cell trap creates spatial separation of single cells before compartmentalization using valves. [Reprinted (adapted) with permission from ref. 14. Copyright 2011 National Academy of Sciences, USA]. B) Example of selective encapsulation of single particles/cells in aqueous droplets [Reprinted (adapted) with permission from ref. 15. Copyright 2005 American Chemical Society]. C) Single cells in 
suspension are manipulated in-flow before droplet generation, resulting in the majority of discrete volumes contain a single-cell. [Adapted with permission from ref. 16]. 
A)

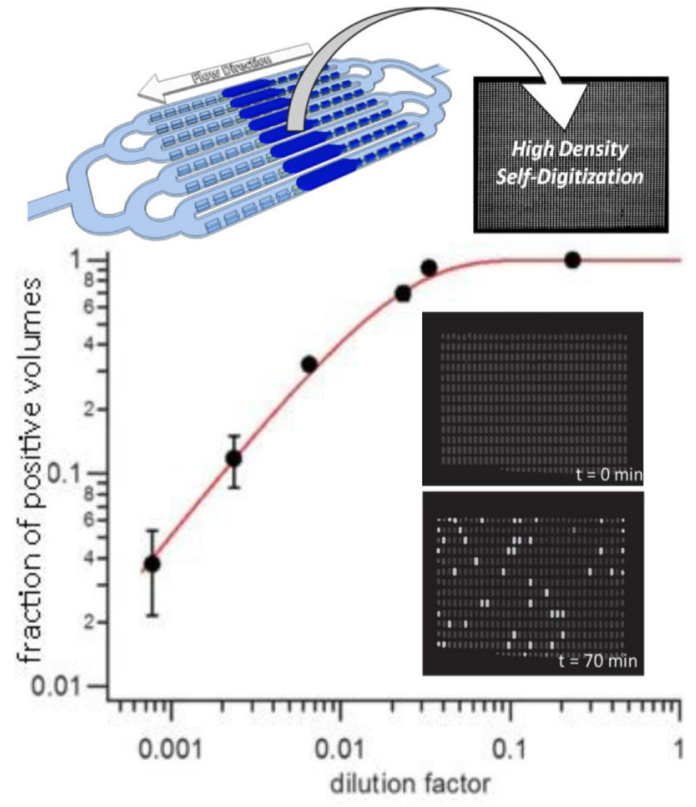

B)
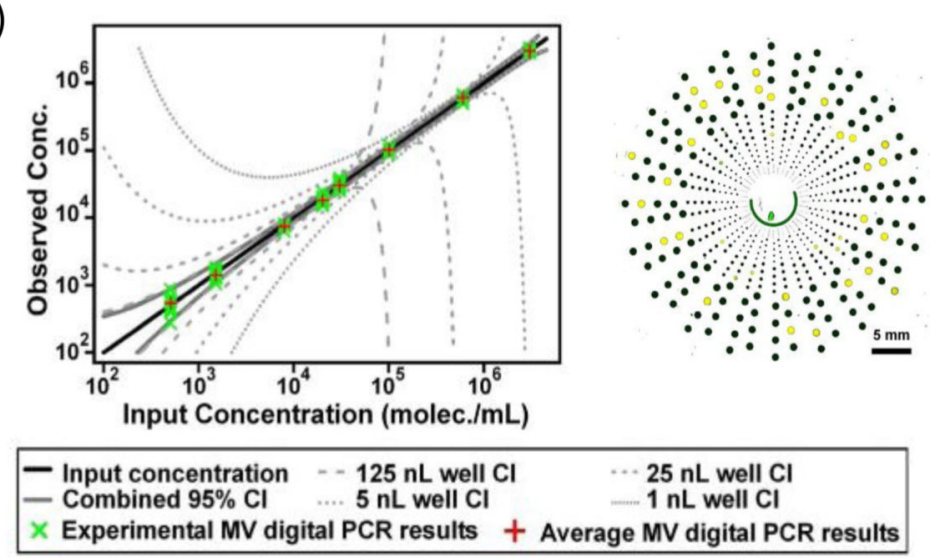

C)
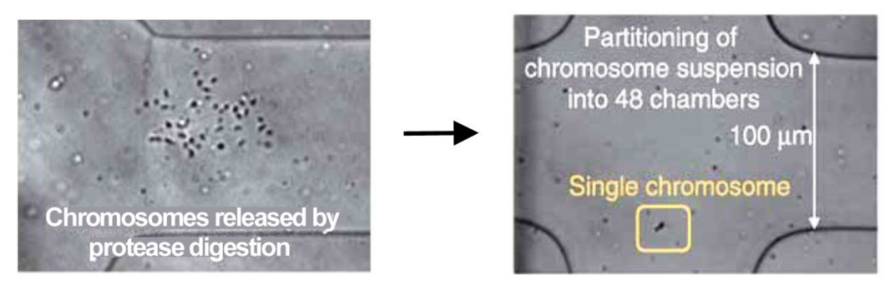

Fig. 4.

Digitization of genetic material for analysis. (A) The genetic material in a sample can be broken into many small volumes before gene-specific amplification. [Reprinted (adapted) with permission from ref. 27.Copyright 2013 American Chemical Society]. Signal accumulation allows for the counting of positive reactions. The fraction of positive volumes correlates to absolute copy number without the need for a reference standard. [Adapted from ref. 28]. (B) Multi-volume (MV) digital PCR can reduce the number of volumes necessary to achieve high dynamic range. [Reprinted (adapted) with permission from ref. 29.Copyright 
2011 American Chemical Society]. (C) Single chromosomes are partitioned to preserve haplotype information through multiple strand displacement and sequencing. [Reprinted (adapted) with permission from ref. 17. Copyright 2011 Nature Publishing Group]. 